\title{
Synthesis, Characterization of Metal-Schiff Base Functionalized Mesoporous Silica for Pesticide Adsorption
}

\author{
Huayao CHEN, Xuemin CHEN, Hongjun ZHOU *, Xinhua ZHOU, Hua XU
} School of Chemistry and Chemical Engineering, Zhongkai University of Agriculture and Engineering, Guangzhou Key Lab
for Efficient Use of Agricultural Chemicals Guangzhou, P.R. China

crossref http://dx.doi.org/10.5755/j01.ms.25.1.19044

Received 14 September 2017; accepted 13 January 2018

\begin{abstract}
The mesoporous silica modified by salicylaldimine was prepared through a co-condensation method. Through the bridge effect from metal ion (copper ion, zinc ion, manganese ion), the model drug-avermectin was supported on the metal-Schiff base functionalized mesoporous silica (M-MCM-41) to form a highly efficient adsorbents for pesticide delivery and removal. The experimental results showed that the sequence in adsorption capacity (AC) for avermectin (AVM) of various mesoporous silica in different adsorption time was Zn-MCM-41 > Cu-MCM-41 > Mn-MCM-41 > MCM-41 > SA-MCM-41. The AC of Zn-MCM-41 was $151 \mathrm{mg} / \mathrm{g}$, while the MCM-41 was $78 \mathrm{mg} / \mathrm{g}$ which increased nearly $100 \%$ after modification due to its strongest coordination ability. The FT-IR and XPS results confirmed the existence of coordination bond between SA-MCM-41 and metal ions as well as the coordination bond between M-MCM-41 and avermectin. The kinetic data and the equilibrium isotherms are modeled by three kinetic models, the pseudo-first-order, the pseudo-second-order and intraparticle diffusion, and two isotherm models, Langmuir and Freundlich, respectively. The kinetic mechanism of the adoption changed from physical adsorption to the intraparticle diffusion due to the stronger interaction between avermectin and the mesoporous silica through coordination. Adsorption isotherm of the samples were well-represented by Freundlich model which indicated that the adsorption was reversible.

Keywords: metal Schiff base, MCM-41, avermectin, adsorption.
\end{abstract}

\section{INTRODUCION}

Nowadays, the materials with highly dispersed pores have drawn significant interests [1]. And the ordered mesoporous materials are most attractive among these materials [2-4] due to their uniform and adjustable pore sizes from $2 \mathrm{~nm}$ to $50 \mathrm{~nm}$ complied with IUPAC recommendation [5] especially for mesoporous silica. The organic templates (i.e., surfactant-types) are usually applied in the synthesis of mesoporous silica materials $[6,7]$. Until now, the mesoporous silica materials have already been applied in many practical areas such as sensors and separations [8], catalysis [9], novel functional materials [10], selective adsorption [11] as well as being a host to guest molecules [12].

These silica materials are potential candidates for sustained-release and adsorption application due to their high thermal stability, easy morphology control, and various surface functionalizations. The sustained released of pharmaceutical active agents and drug delivery has been studied in the literatures $[13,14]$. Inspired by the researches mentioned above, the following studies of using MCM-41 as adsorbent [15] and drug delivery [16] systems have significant growth. The main reason for using mesoporous silica materials for sustained released and adsorption systems was due to their high ordered pore network with high pore volume which provides more space for the quantities of adsorbed samples. What's more, the fine control of the release kinetics and drug load can be achieved by the ordered network with homogeneous size. The MCM-
41 modified with amine groups was also studied for this system and resulted in better release kinetics [17].

The influence of synthesis and surface modification of MCM-41 by aminopropyl groups on the immobilization and subsequent release of acetylsalicylic acid were also studied in several papers $[18,19]$ in which MCM-41 materials were functionalized through the co-condensation method, postsynthesis treatment and solvothermal processes. Meanwhile, the preparation of sustained-release system with $\mathrm{pH}$ sensitivity coordinated with metal ion which acts as a bridge to improve its adsorption and sustained-release performance were also frequently reported [20,21]. However, the study based on MCM-41 coordinated with metal for the pesticide delivery and adsorption was rarely reported.

In our previous research we proposed the $\mathrm{pH}$ responsive chlorpyrifos/copper Schiff base modified mesoporous silica (Cu-MCM-41) sustained-release system, which was prepared by the coordination of copper ions through impregnation method and show a high performance $[22,23]$. But the effect of different metal ion and the mechanism of adsorption had not been investigated yet. In this paper, the Dodecyl trimethyl ammonium bromide (DTAB) was adopted as template and tetraethylorthosilicate (TEOS) as silica source respectively. The self-made salicylaldimine was used as organic modifying agent to prepare salicylaldimine modified mesoporous silica by cocondensation method. Through the bridge effect from metal ion, avermectin (AVM)-a family of macrocyclic lactones used in the control of nematode and arthropod parasites

\footnotetext{
* Corresponding author. Tel.: +86-020-89003114; fax: +86-02089003114. E-mail address: hongjunzhou@163.com (H. Zhou)
} 
which has very poor aqueous solubility and recognized as a nuisance less biological pesticide, was supported on the Schiff base modified mesoporous silica. The relationship between different metal ions with its adsorption performance was also investigated. Finally, the highly efficient adsorbents with high adsorption capacity for pesticide delivery and removal was developed which would thus be expected to increase the efficiency of the earth recovery project from pesticide pollution and decrease pesticide sprayed frequency needed in the agriculture due to the pesticide sustained released.

\section{MATERIALS AND METHODS}

\subsection{Materials}

Dodecyl trimethyl ammonium bromide (DTAB), tetraethyl orthosilicate (TEOS), ethanol, dichloromethane, anhydrous magnesium sulfate,ammonia, zinc nitrate, manganese nitrate, copper nitrate, sodium hydroxide, hydrochloride were obtained from Tianjin Damao Chemical Reagents. 3-aminopropyltriethyloxy silane (APTES), salicylaldehyde were obtained from Aladdin. And avermectin (Hubei Kangsheng Chemical Engineering Co., Ltd.) were also used in this work. All chemicals were analytical grade and used as received without any further purification.

\subsection{Sample preparation and characterization}

According to previous research [22, 23], sol-gel method was adopted to prepare MCM-41. $2.0 \mathrm{~g}$ of DTAB, $100 \mathrm{~mL}$ deionized water and $60 \mathrm{~mL}$ of ammonia were added to the flask to be dissolved at $60^{\circ} \mathrm{C}$ with stirring for $1 \mathrm{~h} .5 \mathrm{~g}$ of TEOS was added to the solution dropwisely for $6 \mathrm{~h}$ before being crystalized at room temperature for 3 days. Then the samples were obtained after filtered, washed and dried. Finally, the template was removed by ethanol to attain MCM-41.

According to the literature [7], $4.42 \mathrm{~g}$ of APTES, $2.44 \mathrm{~g}$ of salicylaldehyde and $100 \mathrm{~mL}$ of ethanol were added into a flask and reacted at $95^{\circ} \mathrm{C}$ for $3 \mathrm{~h}$. Ethanol was removed by rotary evaporated, and $20 \mathrm{~mL}$ of dichloromethane was added, then the products washed with deionized water 3 times. The organic layer was extracted and standing for $12 \mathrm{~h}$. Then the product was filtered to remove dichloromethane to attain salicylaldimine.

According to previous research [24], co-condensation method was adopted to prepare salicylaldimine -modified mesoporous silica. $1.0 \mathrm{~g}$ of DTAB, $100 \mathrm{~mL}$ deionized water and $70 \mathrm{~mL}$ of ammonia were added to the flask to be dissolved at $60{ }^{\circ} \mathrm{C}$ with stirring. And $5 \mathrm{~g}$ of TEOS was added to the solution dropwisely. 1 hour later, $1 \mathrm{~g}$ of as synthesized salicylaldimin was added and kept on reacting for $6 \mathrm{~h}$ before being crystalized at room temperature respectively, filtered, washed and dried. Finally, the template was removed by ethanol to attain SA-MCM-41.

According to previous research [22], $100 \mathrm{~mL}$ of copper nitrate, zinc nitrate, manganese nitrate solution $(1 \mathrm{mmol} / \mathrm{L})$ was added to $200 \mathrm{mg}$ of SA-MCM-41 at $35^{\circ} \mathrm{C}$ under stirring for $24 \mathrm{~h}$. Then M-MCM-41 was attained after being filtered, washed and dried.
According to previous research [22], the supported avermectin was prepared via impregnation. M-MCM-41 was activated under vacuum at $80^{\circ}$ for $6 \mathrm{~h}$. And $100 \mathrm{mg}$ of samples was immersed in $20 \mathrm{~mL}$ of avermectin ethanol solution $(10 \mathrm{mg} / \mathrm{mL})$ at $35^{\circ}$ under stirring for $24 \mathrm{~h}$, then filtered, washed, and dried. The obtained products were denoted as AVM-M-MCM-41.

The samples were analyzed using a Bruker AXS D8 Xray diffractometer (Bruker AXSGmbH, Karlsruhe, Germany) with $\mathrm{Cu}$ radiation $(\lambda=1.5418 \mathrm{~nm})$ and a graphite monochromator at $25^{\circ} \mathrm{C}, 40 \mathrm{kV}$, and $30 \mathrm{~mA}$. The measurements were scanned at $2 \% \mathrm{~min}$ (angular range $2 \theta=$ $0.5 \sim 10^{\circ}$ ) in $0.02^{\circ}$ step size. The structure of the particles was analyzed by a Spectrum 100 Fourier infrared spectrometer (Perkin Elmer Inc., USA) by using the $\mathrm{KBr}$ squash technique. The gold particles were sprayed on the surface of samples under protection of $\mathrm{N}_{2}$ and the samples were characterized by an S4800 scanning electron microscope (Hitachi, Japan) to obtain SEM observation. TEM observation was conducted on a FEI Tecnai G2 F20 transmission electron microscope. X-ray photoelectron spectra (XPS) were recorded on a ESCALAB 250XI spectrometer (Thermo Fisher Scientific, Al $\mathrm{K} \alpha$, $\mathrm{h} v=1486.6 \mathrm{eV}, \quad \pm 0.05 \mathrm{eV}$ ) under a vacuum of $\sim 2 \times 10^{-7} \mathrm{~Pa}$. Charging effects were corrected by adjusting the main $\mathrm{C} 1 \mathrm{~s}$ peak to a position of $284.8 \mathrm{eV}$.

\subsection{Adsorption properties test}

A UV-2550 UV-Vis spectrophotometer from Shimadzu Co., Japan, was applied to measure the amount of avermectin adsorbed by mesoporous silica. Linear regression of the solution concentration (C) and absorbance (A) of avermectin standard solutions of different concentrations at $\lambda=245 \mathrm{~nm}$ was performed to obtain a standard curvilinear equation: $\quad \mathrm{C}=26.647 \mathrm{~A}+2.7776$, $\mathrm{R}^{2}=0.9782$. UV spectroscopy was performed to measure the absorbance of this solution before and after the adsorption in avermectin ethanol solution. Adsorption capacity (AC) may be calculated by the following equation:

$A C=\frac{\left(C_{o}-C_{1}\right) \times V}{m}$

where $C_{0}$ is the origin mass concentration $(\mathrm{mg} / \mathrm{L})$ of the avermectin in ethanol solution, $C_{l}$ is the mass concentration $(\mathrm{mg} / \mathrm{L})$ of the avermectin in ethanol solution after adsorption, and $\mathrm{m}$ is the mass ( $\mathrm{g}$ ) of mesoporous silica.

\subsection{Kinetic and thermodynamics study}

Adsorption kinetics that describes the rate of an adsorbate adsorption on an adsorbent is useful for designing of waste water treatment systems. These models are pseudofirst-order (Eq. 2), pseudo-second-order (Eq. 3) and intraparticle diffusion (Eq. 4) kinetic models. The linearized forms of these equations are expressed as below [25]:

$$
\begin{aligned}
& \log \left(q_{e}-q_{t}\right)=\log q_{e}-\frac{k_{1} t}{2.303} ; \\
& \frac{\mathrm{t}}{q_{t}}=\frac{x}{k_{2} q_{e}^{2}}+\frac{\mathrm{t}}{q_{e}} ; \\
& q_{t}=k_{3} t^{0.5}+C,
\end{aligned}
$$


where $q_{e}$ and $q_{t}$ are the experimental amount of AVM adsorbed by mesoporous silica $(\mathrm{mg} / \mathrm{g})$ at equilibrium and at time $t$, respectively. $k_{1}\left(\mathrm{~min}^{-1}\right), k_{2}(\mathrm{~g} / \mathrm{mg} \cdot \mathrm{min})$ and $k_{3}$ $\left(\mathrm{mg} / \mathrm{g} \cdot \mathrm{min}^{0.5}\right)$ are the pseudo-first-order, the pseudo-secondorder and the intraparticle diffusion rate constants, respectively. Rate constants $k_{1}$ is calculated from the slopes of the linear plots of $\ln \left(q_{e}-q_{t}\right)$ versus $t$, while $k_{2}$ is calculated from the intercepts of the linear plots of $t / q_{t}$ versus $t$.

Two-parameter isotherm models, namely, Langmuir and Freundlich are used to describe the adsorption isotherms of MCM-41, SA-MCM-41, Zn-MCM-41, Cu-MCM-41 and Mn-MCM-41. The linearized forms of Langmuir and Freundlich models are given by Eq. 5 and Eq. 6 , respectively [26]:

$\frac{C_{e}}{q_{e}}=\frac{C_{e}}{Q_{m}}+\frac{1}{Q_{m} b}$

$\ln q_{e}=\ln K+\frac{1}{n} \ln C$,

where $C_{e}$ is the avermectin concentration in the liquid-phase $(\mathrm{mg} / \mathrm{L}), q_{e}$ is the amount of avermectin adsorbed by mesoporous silica at equilibrium $(\mathrm{mg} / \mathrm{g})$ and $Q_{m}$ is the maximum adsorption capacity of mesoporous silica $(\mathrm{mg} / \mathrm{g})$. The Langmuir $(b, \mathrm{~L} / \mathrm{mg})$ and Freundlich $\left(K, \mathrm{mg}^{1-\mathrm{n}} \mathrm{L}^{\mathrm{n}} / \mathrm{g}\right)$ isotherm constants are related to the adsorption energy and the adsorption capacity, respectively. The constant $n$ (unitless) is related to the adsorption intensity. Langmuir and Freundlich isotherm parameters are calculated from the slope and intercept values of linear plots of $C_{e} / q_{e}$ versus $C_{e}$ and $\log q_{e}$ versus $\log C_{e}$, respectively.

\section{RESULTS AND DISCUSSION}

\subsection{Characterization of mesoporous materials}

Fig. 1 showes the XRD patterns of MCM-41, SAMCM-41 and Zn-MCM-41. There were two characteristic peaks shown in MCM-41, which could be ascribed to (100), and (200) crystal face respectively, which indicated that the particles had regular hexagonal pore structure [23, 27].

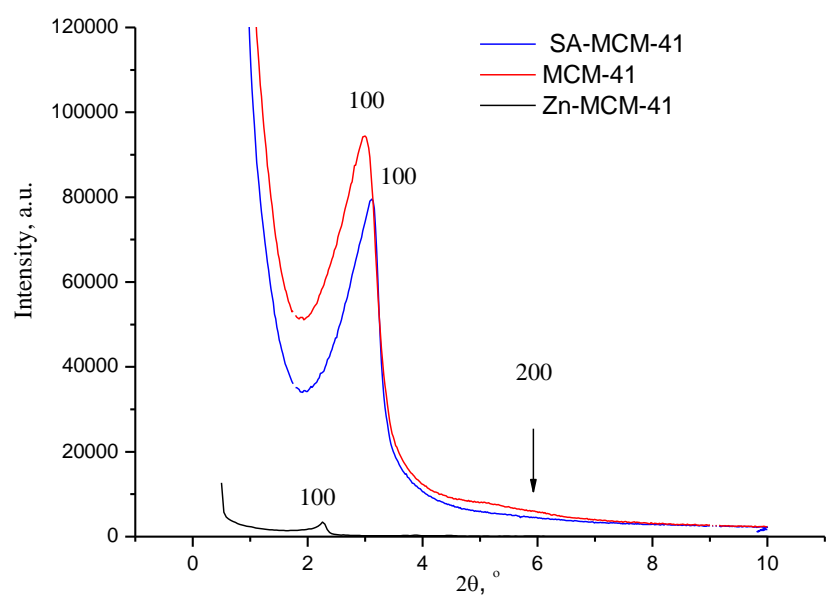

Fig. 1. XRD patterns of MCM-41, SA-MCM-41 and Zn-MCM-41

As modified by salicylaldimine, the strength of the XRD peaks decreased, which proved that APTES was introduced to the system and decreased its degree of orderliness $[23,28]$. The XRD peaks of the sample with metal ion has a notable negative shift proving that the lattice distortion happened due to the cooperation interaction between metal ion and MCM-41. And the loading of metal ion further decreased its degree of orderliness due to the block of the pores by metal salts.

Fig. 2 depicted the SEM and TEM image of MCM-41 $(\mathrm{a} \& \mathrm{~b})$ and SA-MCM-41 (c\&d). As shown, the surface of the particles presented more rough after salicylaldimine modification. And the regular hexagonal pore structure was well-maintained without agglomeration after which was in consistent with the XRD results.

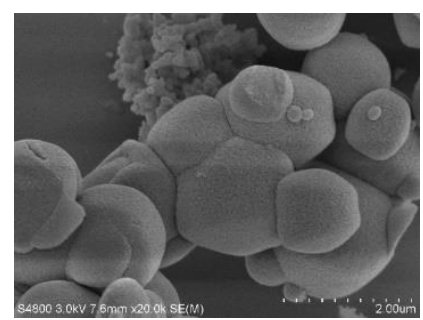

a

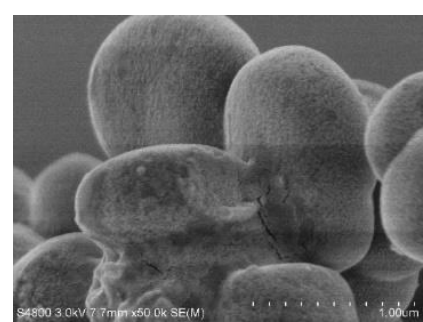

c

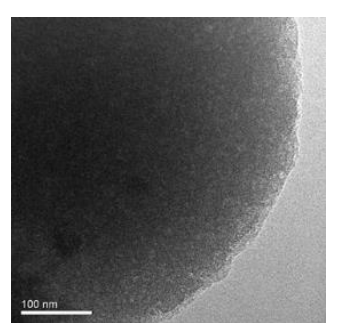

b

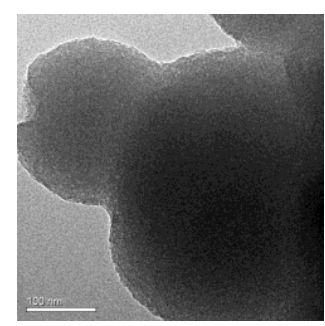

d
Fig. 2. SEM and TEM image of MCM-41 ( $a$ and $b$ ) and SA-MCM$41(\mathrm{c}$ and $\mathrm{d})$

FTIR was carried out to compare the different composition of MCM-41, SA-MCM-41, Zn-MCM-41, CuMCM-41 and Mn-MCM-41. As shown in Fig. 3, two bands appeared in $3420 \mathrm{~cm}^{-1}$ and $960 \mathrm{~cm}^{-1}$ for MCM-41 ascribed to stretching and bending vibration of $\mathrm{Si}-\mathrm{OH}$ respectively $[23,29]$.



Fig. 3. FTIR spectra of MCM-41, SA-MCM-41, Zn-MCM-41, Mn-MCM-41 and Cu-MCM-41

Two bands appeared in $2847 \mathrm{~cm}^{-1}$ and $2936 \mathrm{~cm}^{-1}$ were ascribed to the characteristic peaks of methylene group due to the surfactant residue added during preparation. $1081 \mathrm{~cm}^{-}$ ${ }^{1}$ and $800 \mathrm{~cm}^{-1}$ were attributed to the characteristic peaks of $\mathrm{Si}-\mathrm{O}-\mathrm{Si}$ on the $\mathrm{SiO}_{2}$ framework. Comparing to MCM-41, a 
new band appeared at $1631 \mathrm{~cm}^{-1}$ belonging to the stretching band of $\mathrm{C}=\mathrm{N}$ and the vibration band of benzene ring in the salicylaldimine. The blue shift of $\mathrm{C}=\mathrm{N}$ from $1631 \mathrm{~cm}^{-1}$ to $1641 \mathrm{~cm}^{-1}$ happened after the coordination with metal ion.

The XPS analysis was carried out to identify the surface elements chemical states, as shown in Fig. 4.
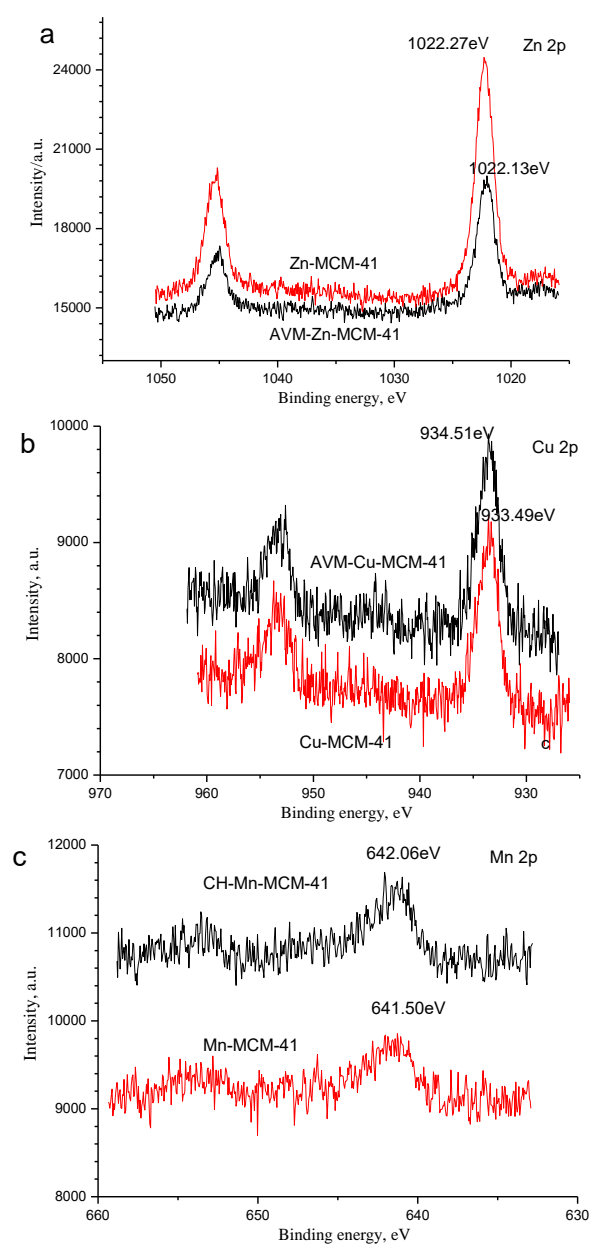

Fig. 4. a - XPS spectra of Zn-MCM-41 and AVM-Zn-MCM-41; $\mathrm{b}-\mathrm{Cu}-\mathrm{MCM}-41$ and AVM-Cu-MCM-41; c-Mn-MCM-41 and AVM-Mn-MCM-41

After loading avermectin, the binding energy (BE) of zinc ion decreased from $1022.27 \mathrm{eV}$ to $1022.13 \mathrm{eV}$. While for $\mathrm{Cu}-\mathrm{MCM}-41$ and $\mathrm{Mn}-\mathrm{MCM}-41$, the binding energy (BE) of metal ion increased from $933.49 \mathrm{eV}$ to $934.51 \mathrm{eV}$ and $641.50 \mathrm{eV}$ to $642.06 \mathrm{eV}$ respectively after loading avermectin which implies that their coordination interaction was weaker after loading AVM in contrary to Zn-MCM-41. The results above also confirmed the coordination interaction between metal ion and avermectin $[29,30]$.

\subsection{Adsorption performance}

Fig. 5 a showed the adsorption performance of various mesoporous silica in different adsorption time with a sequence of Zn-MCM-41 > Cu-MCM-41 > Mn-MCM$41>$ MCM-41 > SA-MCM-41 in adsorption capacity. The AC decreased because of the pores blocked after modified by salicylaldimine, and then increased significantly after coordinated with metal ion, which improved the interaction between avermectin and mesoporous silica support through coordination [21]. Among them, Zn-MCM-41 has the biggest $\mathrm{AC}(151 \mathrm{mg} / \mathrm{g})$ due to its strongest coordination ability in accordance with the XPS results among the three coordinated metal ion.
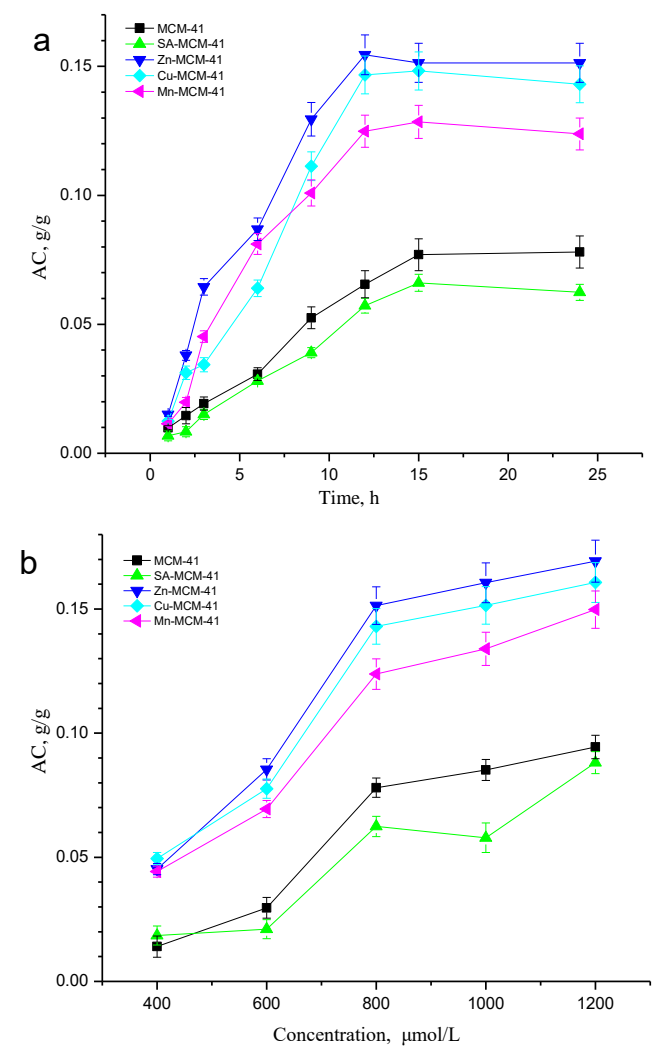

Fig. 5. $a-$ the AC of mesoporous silica in different time; $b-A V M$ concentration

As shown in Fig. 5b, the AC of the mesoporous silica increased while the avermectin concentration of the solution increased during the adsorption with the same $\mathrm{AC}$ sequence of Fig. 5 a. When the avermectin concentration of the solution was between $600 \mu \mathrm{mol} / \mathrm{L}$ to $800 \mu \mathrm{mol} / \mathrm{L}$, the increased of the AC was fastest. While the avermectin concentration further increased from $800 \mu \mathrm{mol} / \mathrm{L}$ to $1200 \mu \mathrm{mol} / \mathrm{L}$, the increased speed of AC slowed down and maintained steady above $1200 \mu \mathrm{mol} / \mathrm{L}$.

\subsection{Kinetics and thermodynamics study}

From the slope and intercept values of linear fittings of the experimental kinetics of avermectin onto mesoporous silica to the aforementioned kinetic models, the kinetic parameters are calculated and the data obtained are tabulated in Table 1. Additionally, the determination coefficient values, $R^{2}$, of the plots are also given in this table. From the data of this table, it can be observed that linear fittings of the experimental kinetic data of MCM-41 to the pseudo-first-order kinetic model resulted in higher determination coefficient values $\left(R^{2}=0.926\right)$ than their fittings to the pseudo-second-order model $\left(R^{2}=0.550\right)$ and intraparticle diffusion model $\left(R^{2}=0.866\right)$ proving that the physical adsorption is the main process for the mesoporous silica without modification. For applicability of the intraparticle diffusion kinetic model to the present adsorption processes, the plot of $q_{t}$ versus $t^{0.5}$ must yield straight lines passing through the origin. 
Table 1. Kinetic model of MCM-41, SA-MCM-41, Zn-MCM-41, Cu-MCM-41 and Mn-MCM-41 fitting results for drug adsorption curves of avermectin-loaded particles

\begin{tabular}{|c|c|c|c|c|c|c|}
\hline \multirow{2}{*}{ Sample } & \multicolumn{2}{|c|}{ Pseudo-first-order } & \multicolumn{2}{|c|}{ Pseudo-second-order } & \multicolumn{2}{|c|}{ Intraparticle diffusion } \\
\hline & $k_{1}$ & $R^{2}$ & $k_{2}$ & $R^{2}$ & $k_{3}$ & $R^{2}$ \\
\hline MCM-41 & 0.156 & 0.926 & 0.153 & 0.550 & 0.262 & 0.866 \\
\hline SA-MCM-41 & 0.159 & 0.873 & 0.169 & 0.435 & 0.018 & 0.917 \\
\hline Zn-MCM-41 & 0.215 & 0.930 & 0.078 & 0.266 & 0.844 & 0.989 \\
\hline Cu-MCM-41 & 0.350 & 0.730 & 0.109 & 0.357 & 0.041 & 0.887 \\
\hline Mn-MCM-41 & 0.288 & 0.880 & 0.122 & 0.332 & 0.476 & 0.985 \\
\hline
\end{tabular}

The results obtained in this study illustrate that straight lines are obtained for the intraparticle diffusion model for MCM-41 after modification. $R^{2}$ were $0.917,0.989,0.887$ and 0.985 for SA-MCM-41, Zn-MCM-41, Cu-MCM-41 and Mn-MCM-41 respectively which were much higher than the other two models. This implies that the intraparticle diffusion model is appropriate to fit the kinetic data of avermectin onto modified mesoporous silica and is therefore the rate-controlling step in the present adsorption processes [31].

Langmuir and Freundlich isotherm parameters are calculated from the slope and intercept values of linear plots of $C_{e} / q_{e}$ versus $C_{e}$ and $\log q_{e}$ versus $\log C_{e}$, respectively. The isotherm parameters obtained for both models are given in Table 2 together with the determination coefficient values, $R^{2}$. The $R^{2}$ values of this table suggest that the equilibrium data are well-described by Freundlich model than Langmuir mode which implied that their adsorption is reversible.

Table 2. Thermodynamic model of MCM-41, SA-MCM-41, ZnMCM-41, Cu-MCM-41 and Mn-MCM-41 fitting results for drug adsorption curves of avermectin-loaded particles

\begin{tabular}{|l|c|c|c|c|}
\hline \multirow{2}{*}{ Sample } & \multicolumn{2}{|c|}{ Langmuir model } & \multicolumn{2}{c|}{ Freundlish model } \\
\cline { 2 - 5 } & $b$ & $R^{2}$ & $K$ & $R^{2}$ \\
\hline MCM-41 & -0.0006 & 0.582 & $10^{-8.4}$ & 0.913 \\
\hline SA-MCM-41 & -0.0004 & 0.261 & $10^{-6.3}$ & 0.834 \\
\hline Zn-MCM-41 & -0.0002 & 0.024 & $10^{-3.6}$ & 0.904 \\
\hline Cu-MCM-41 & -0.0001 & 0.146 & $10^{-2.9}$ & 0.911 \\
\hline Mn-MCM-41 & -0.0001 & 0.006 & $10^{-3.2}$ & 0.940 \\
\hline
\end{tabular}

Because the former model has higher $R^{2}$ values $(0.913$, $0.834,0.904,0.911,0.940$ for MCM-41, SA-MCM-41, ZnMCM-41, Cu-MCM-41 and Mn-MCM-41) than the later one $(0.582,0.261,0.024,0.146$ and 0.006 for MCM-41, SAMCM-41, Zn-MCM-41, Cu-MCM-41 and Mn-MCM-41.

By now, the complete drug adsorption of M-MCM-41 was generally illustrated in consideration of kinetic and thermodynamics mechanism and the data in this work. Metal ion act as a bridge to coordinate with Schiff base modified mesoporous silica and avermectin. The kinetic mechanism of the adoption changed from physical adsorption to the intraparticle diffusion inducing the importance of pore effect on the adsorption process was significantly enhanced due to the stronger interaction between avermectin and the modified mesoporous silica.

\section{CONCLUSIONS}

In conclusion, the salicylaldimine modified mesoporous silica (SA-MCM-41) was prepared by cocondensation method. Metal-Schiff base mesoporous silica was prepared through coordination between metal ion and Schiff base. The characterization confirmed the existence of coordination bond between MCM-41 and metal ions and between M-MCM-41 and avermectin. The AC of Zn-MCM41 was $151 \mathrm{mg} / \mathrm{g}$, while the MCM-41 was $78 \mathrm{mg} / \mathrm{g}$ which increased nearly $100 \%$ after modification due to its strongest coordination ability in accordance with the XPS results among the three coordinated metal ions. After modified by salicylaldimine and metal ions, the kinetic mechanism of the mesoporous silica adoption changed from physical adsorption to the intraparticle diffusion due to the stronger interaction between avermectin and the mesoporous silica through coordination. Adsorption isotherm of the samples was well-represented by Freundlich model which implied that their adsorption is reversible.

\section{Acknowledgments}

This research was supported by National Natural Science Foundation of China (Grant No. 21606262), Natural Science Foundation of Guangdong Province (Grant No. 2017A030311003), Science and Technology Program of Huadu district (Grant No. HD15CXY002), Science and Technology Program of Guangzhou, China (Grant No.201707010473), Ordinary University Innovation Project of Guangdong Province (Grant No. 2016KTSCX062).

\section{REFERENCES}

1. Chen, D., Caruso, R.A. Recent Progress in the Synthesis of Spherical Titania Nanostructures and their Applications Advanced Functional Materials 23 (11) 2013: pp. $1356-1374$. https://doi.org/10.1002/adfm.201201880

2. Schmack, R., Eckhardt, B., Koch, G., Ortel, E., Kraehnert, R. ZnO Coatings with Controlled Pore Size, Crystallinity and Electrical Conductivity Materials Science (Medžiagotyra) 22 (1) 2016: pp. 74-81. http://dx.doi.org/10.5755/j01.ms.22.1.8634

3. Cao, W., Hu, S.S., Ye, L.H., Cao, J. Dispersive Micro-solidphase Extraction Using Mesoporous Hybrid Materials for Simultaneous Determination of Semivolatile Compounds from Plant Tea by Ultra-high-performance Liquid Chromatography Coupled with Quadrupole Time-of-flight Tandem Mass Spectrometer Journal of Agricultural and Food Chemistry 62 (40) 2014: pp. 9683-9689. http://dx.doi.org/10.1021/jf5029625

4. Liang, Z., Shi, W., Zhao, Z., Sun, T., Cui, F. Enhanced Removal and Adsorption Characters of Aniline by the Inorganically Modified Mesoporous Silica Nano-spheres Colloids and Surface. A-Physicochemical and Engineering Aspects 513 2017: pp. 250-258. 
https://doi.org/10.1016/j.colsurfa.2016.10.050

5. Burness, L.T. Mesoporous Materials: Properties, Preparation and Application. Nova Science Publishers, UK, 2009: pp. 1-34.

6. Blin, J.L., Michaux, F., Stébé, M.J. Nanostuctured Mesoporous Materials from Different Silica Sources Using Fluorinated Surfactants as Templates Colloids and Surface. A-Physicochemical and Engineering Aspects 510 2016: pp. $104-112$. https://doi.org/10.1016/j.colsurfa.2016.04.067

7. Kresge, C.T., Leonowicz, M.E., Roth, W.J., Vartuli, J., Beck, J. Ordered Mesoporous Molecular Sieves Synthesized by a Liquid-crystal Template Mechanism Nature 359 (6397) 1992: pp. $710-712$.

https://doi.org/10.1038/359710a0

8. Thakur, S.S., Chauhan, G.S. Gelatin-silica-based Hybrid Materials as Efficient Candidates for Removal of Chromium(VI) from Aqueous Solutions Industrial \& Engineering Chemistry Research 53 (12) 2014: pp. $4838-4849$.

https://doi.org/10.1021/ie401997g

9. Vargas-Hernández, D., Rubio-Caballero, J.M., Santamaría-González, J., Moreno-Tost, R., MéridaRoblesb, J.M., Pérez-Cruza, M.A., Jiménez-Lópezb, A., Hernández-Huescaa, R., Maireles-Torresb, P. Furfuryl Alcohol from Furfural Hydrogenation over Copper Supported on SBA-15 Silica Catalysts Journal of Molecular Catalysis A: Chemical 383-384 (3) 2014: pp. 106-113. https://doi.org/10.1016/j.molcata.2013.11.034

10. Bajare, D., Bumanis, G., Korjakins, A. New Porous Material Made from Industrial and Municipal Waste for Building Application Materials Science 20 (3) 2014: pp. $333-338$.

http://dx.doi.org/10.5755/j01.ms.20.3.4330

11. Zhang, Z., Hou, X., Zhang, X., Li, H. The Synergistic Adsorption of Pyrene and Copper onto Fe(III) Functionalized Mesoporous Silica from Aqueous Solution Colloids and Surface. A-Physicochemical and Engineering Aspects 520 2017: pp. $39-45$.

https://doi.org/10.1016/j.colsurfa.2017.01.056

12. Knežević, N.Z., Durand, J.O. Large Pore Mesoporous Silica Nanomaterials for Application in Delivery of Biomolecules Nanoscale 7 (6) 2015: pp. 2199-2209.

https://doi.org/10.1039/c4nr06114d

13. Wibowo, D., Zhao, C.X., Peters, B.C., Anton, P.J.M. Sustained Release of Fipronil Insecticide in Vitro and in Vivo from Biocompatible Silica Nanocapsules Journal of Agricultural and Food Chemistry 62 (52) 2014: pp. $12504-12511$.

https://doi.org/10.1021/jf504455x

14. An, J., Zhang, X., Guo, Q., Zhao, Y., Wu, Z., Li, C. Glycopolymer Modified Magnetic Mesoporous Silica Nanoparticles for Mr Imaging and Targeted Drug Delivery Colloids and Surface. A-Physicochemical and Engineering Aspects 482 2015: pp. 98-108.

https://doi.org/10.1016/j.colsurfa.2015.04.035

15. Intana, T., Föttinger, K., $\quad$ Rupprechter, G., Kongkachuichay, P. Physicochemical Properties of $\mathrm{Cu}$ Loaded onto Core-shell Al-MCM-41: Effect of Loading Methods Colloids and Surface. A-Physicochemical and Engineering Aspects 467 2015: pp. 157-165. https://doi.org/10.1016/j.colsurfa.2014.11.048

16. Arruebo, M. Drug Delivery from Structured Porous Inorganic Materials Wiley Interdiscience Review Nanomedical and Nanobiotechnology 4 (1)
2012: pp. $16-30$.

https://doi.org/10.1002/wnan.132

17. Rámila, A., Muñoz, B., Pérez-Pariente, J., Vallet-Regí, J. Mesoporous MCM-41 as Drug Host System Journal of Sol-Gel Science and Technology 26(1-3) 2003: pp. $1199-1202$.

https://doi.org/10.1023/A:1020764319963

18. Gao, L., Sun, J., Li, Y. Functionalized Bimodal Mesoporous Silicas as Carriers for Controlled Aspirin Delivery Journal of Solid State Chemistry 184 (8) 2011: pp. 1909-1914. https://doi.org/10.1016/j.jssc.2011.05.052

19. Datt, A., El-Maazawi, I., Larsen, S. C. Aspirin Loading and Release from MCM-41 Functionalized with Aminopropyl Groups via Co-condensation or Postsynthesis Modification Methods The Journal of Physical Chemistry C 116 (34) 2012: pp. $18358-18366$.

https://doi.org/10.1021/jp3063959

20. Liang, H., Zhou, B., Li, J., Xu, W., Liu, S., Li, Y., Chen, Y., Li, B. Superamolecular Design of Coordination Bonding Architecture on Zein Nanoparticles for $\mathrm{pH}$-responsive Anticancer Drug Delivery Colloids Surfurfaces B: Biointerfaces 136 2015: pp. 1224-1233. https://doi.org/10.1016/j.colsurfb.2015.09.037

21. Zheng, H., Wang, Y., Che, S. Coordination Bonding-Based Mesoporous Silica for $\mathrm{pH}$-responsive Anticancer Drug Doxorubicin Delivery The Journal of Physical Chemistry C 115 (34) 2011: pp. 16803-16813. https://doi.org/10.1021/jp203799m

22. Chen, H., Lin, Y., Zhou, H., Zhou, X., Gong, S., Xu, H. Synthesis, Characterization of Chlorpyrifos/Copper(ii) Schiff Base Mesoporous Silica with $\mathrm{pH}$-sensitivity for Pesticide Sustained Released Journal of Agricultural and Food Chemistry 64 (43) 2016: pp. 8095-8102. https://doi.org/10.1021/acs.jafc.6b03262

23. Chen, H., Lin, Y., Zhou, H., Zhou, X., Gong, S., Xu, H. Highly Efficient Alginate Sodium Encapsulated Chlorpyrifos/Copper(II) Schiff Base Mesoporous Silica Sustained Release System with $\mathrm{pH}$ and ion Response for Pesticide Delivery RSC Advances 6(115) 2016: pp. $114714-114721$. https://doi.org/10.1039/C6RA23836J

24. Shahnaz, R., Masoomeh, N. An Environmentally Benign Multicomponent Synthesis of Some Novel 2-Methylthio Pyrimidine Derivatives Using MCM-41- $\mathrm{NH}_{2}$ as Nanoreactor and Nanocatalyst Journal of Heterocyclic Chemistry 51 (2) 2014: pp. $418-422$.

https://doi.org/10.1002/jhet.1755

25. Dolatyari, L., Yaftian, M.R., Rostamnia, S. Adsorption Characteristics of $\mathrm{Eu}(\mathrm{III})$ and $\mathrm{Th}(\mathrm{IV})$ Ions onto Modified Mesoporous Silica SBA-15 Materials Journal Taiwan Institution Chemical Engineering 60 2016: pp. 174-184. https://doi.org/10.1016/j.jtice.2015.11.004

26. Zhang, H., Wang, X., Liang, H., Tan, T., Wu, W. Adsorption Behavior of Th(IV) onto IIllite: Effect of Contact Time, $\mathrm{pH}$ Value, Ionic Strength, Humic Acid and Temperature Applied Clay Science 127-128 2016: pp. 35-43. https://doi.org/10.1016/j.clay.2016.03.038

27. Kang, J., Park, J., Kim, J.H., Lee, C.G., Kim, S.B. Surface Functionalization of Mesoporous Silica MCM-41 with 3aminopropyltrimethoxysilane for Dye Removal: Kinetic, Equilibrium, and Thermodynamic Studies Desalination and Water Treatment 57 (15) 2016: pp. 7066-7078. https://doi.org/10.1080/19443994.2015.1014856

28. Hartono, S.B., Qiao, S.Z., Jack, K., Ladewig, B.P., Hao, Z., Lu, G.Q. Improving Adsorbent Properties of Cage-like 
Ordered Amine Functionalized Mesoporous Silica with Very Large Pores for Bioadsorption Langmuir 25 (11) 2009: pp. $6413-6424$.

https://doi.org/10.1021/la900023p

29. He, L., Huang, Y., Zhu, H., Zheng, W., Wong, Y., Chen, T. Cancer-targeted Monodisperse Mesoporous Silica Nanoparticles as Carrier of Ruthenium Polypyridyl Complexes to Enhance Theranostic Effects Advanced Functional Materials 24 (19) 2014: pp. 2754-2763. https://doi.org/10.1002/adfm.201303533

30. Li, Y., Shubina, T.E., Chen, M., Shi, Z., Schmid, M., Sterinruck, H.P., Gottfried, J.M., Lin, N. Coordination and
Metalation Bifunctionality of $\mathrm{Cu}$ with 5,10,15,20-tetra(4pyridyl)porphyrin: Toward a Mixed-valence Twodimensional Coordination Network Journal of American Chemistry Society 134 (14) 2012: pp. 6401-6408. https://doi.org/10.1021/ja300593w

31. Mahmoud, M.R., Rashad, G.M., Metwally, E., Saad, E.A., Elewa, A.M. Adsorptive Removal of $134 \mathrm{Cs}^{+}, 60 \mathrm{Co}^{2+}$, and $152+154 \mathrm{Eu}^{3+}$, Radionuclides from Aqueous Solutions Using Sepiolite: Single and Multi-Component Systems Applied Clay Science 141 2017: pp. $72-80$.

https://doi.org/10.1016/j.clay.2016.12.021 